\title{
小菜蛾性信息素微胶囊的合成及其田间诱捕 和迷向活性研究
}

\author{
陈增良 ${ }^{\circledR \otimes}$ 方宇凌 ${ }^{\circledR}$ 张钟宁 $\oplus^{*}$ \\ (1) 农业虫鼠害综合治理国家重点实验室, 中国科学院动物研究所, 北京 100080; (2) 中国科学院研究生院, 北京 100049. \\ *联系人, E-mail: zhangzn@ioz.ac.cn)
}

\begin{abstract}
摘要 用明胶和阿拉伯树胶为壁材, 通过复合凝聚法合成了小菜蛾性信息素微胶囊, 测定了其在田间 的释放保留率, 并初步探讨了其对小菜蛾的引诱和迷向活性. 结果表明, 小菜蛾性信息素微胶囊具有一 定的缓释功能, 在相同的性信息素浓度下, 微胶囊的引诱活性明显好于橡胶诱芯. 在迷向实验中, 迷向 田的小菜蛾诱捕数量明显少于化学防治对照田, 说明迷向活性良好. 因此, 小菜蛾性信息素微胶囊的合 成为应用性信息素治理小菜蛾提供了一种新的施用剂型.
\end{abstract}

关键词 小菜蛾 性信息素微胶囊 合成 诱捕 迷向

小菜蛾(Plutella xylostella (L.))属鳞翅目菜蛾科, 是为害十字花科蔬菜的世界性害虫 ${ }^{[1]}$. 国内外已有很 多关于利用小菜蛾性信息素替代传统化学杀虫剂治理 小菜蛾的报道，如利用小菜蛾性信息素进行虫口密度 监测 ${ }^{[2 \sim 5]}$ 、大量诱捕 ${ }^{[1,6 \sim 9]}$ 和迷向 $\stackrel{[10 \sim 15]}{ }$. 小菜蛾性信息素 的主要成分包括顺-11-十六碳烯醛(Z11-16：Ald)、顺11-十六碳烯乙酸酯(Z11-16:Ac)和顺-11-十六碳烯醇

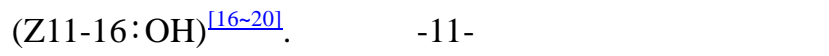
稳定, 易被空气氧化 ${ }^{[21]}$. 空心纤维、橡胶诱芯和石蜡 油是目前应用普遍的昆虫性信息素释放载体 ${ }^{[22]}$. 国外 已有利用性信息素微胶囊作为缓释剂型进行舞毒

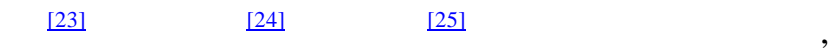
但还未见有关小菜蛾性信息素微胶囊的合成及其应用 效果的相关研究成果. 目前, 昆虫信息素微胶囊的合 成方法主要为复合凝聚法和界面聚合法. 醛在界面聚 合的过程中以及咜存期间均能与脂肪族多胺发生化学 反应, 因此, 醛类信息素不能通过以脂肪族多胺作为 单体的界面聚合法进行微胶囊化; 而醇类信息素亦不 能通过界面聚合法进行微胶囊化, 因为醇能迅速的与 亲电子的单体发生化学反应 ${ }^{[26]}$. 考虑上述因素, 我们 采用复合凝聚法对小菜蛾性信息素进行微胶囊化.

本文对小菜蛾性信息素微胶囊的合成过程及其 在田间的引诱活性和迷向活性进行了研究.

\section{1 材料与方法}

(i ) 材料. 明胶(等电点为 $\mathrm{pH}$ 8.0)和阿拉伯树胶
购自 ACROS 公司(美国); 单宁酸(98\%)为天津福晨 化学试剂厂产品; 戊二醛(25\%)、聚乙烯醇(PVA, 87\%, $100000 \mathrm{Da})$ 和异丙醇为北京盖利精细化学品有限责 任公司产品; 氢氧化钠 $(0.05 \mathrm{~mol} / \mathrm{L})$ 和乙酸 $(0.05$ $\mathrm{mol} / \mathrm{L}$ )购自北京北化精细化学品有限责任公司; 顺11-十六碳烯醛(98\%)、顺-11-十六碳烯乙酸酯(98\%) 和顺-11-十六碳烯乙酸酯(97\%)为东京化成工业株式 会社(日本)产品; 滑石粉 $(<30 \mu \mathrm{m})$ 和橡胶诱芯(绿色, $1.5 \mathrm{~cm} \times 0.8 \mathrm{~cm})$ 为中国科学院动物研究所提供.

(ii) 微胶囊合成过程. 在 2 个 $200 \mathrm{~mL}$ 烧杯中分别 放入 $2.5 \mathrm{~g}$ 阿拉伯树胶和 $2.5 \mathrm{~g}$ 明胶, 各加入 $100 \mathrm{~mL}$ 蒸 馏水, $(20 \pm 1)^{\circ} \mathrm{C}$ 静置 $30 \mathrm{~min}$. 将烧杯放入 $(50 \pm 1)^{\circ} \mathrm{C}$ 的 水浴锅中加热, 并缓慢搅拌至明胶和阿拉伯树胶完全 溶解后倾入 $500 \mathrm{~mL}$ 的三口烧瓶中, 依次加入 $4 \mathrm{~mL}$ (5 $\mathrm{mg} / \mathrm{mL}$ )小菜蛾性信息素正己烷溶液 (Z11-16:Ald, Z11-16:Ac 和 Z11-16:OH, 50:50:1)、20 mL 聚乙烯醇 水溶液(1\%)和明胶水溶液. 将三口烧瓶置于电磁搅拌 器上搅拌(500 r/min) $20 \mathrm{~min}$, 逐滴加入 $10 \%$ 乙酸水溶 液调 $\mathrm{pH} 4.3$ 以引发凝聚形成微胶囊, 并保持搅拌(500 $\mathrm{r} / \mathrm{min}$ ) $20 \mathrm{~min}$. 在此过程中加入 2 滴 Tween-20 以消除 泡沫. $30 \mathrm{~min}$ 后加入 $2 \mathrm{~g}$ 滑石粉(固体分散剂), 随后加 入 $10 \mathrm{~mL} 25 \%$ 的戊二醛引发交联和固化反应. 保持缓 慢搅拌(500 r/min) $1 \mathrm{~h}$ 后, 将三口烧瓶在冰水浴中冷却 至 $5^{\circ} \mathrm{C}$, 逐滴加入 $10 \%$ 氢氧化钠水溶液调 $\mathrm{pH} 9.0$, 再将 三口烧瓶加热到 $25^{\circ} \mathrm{C}$, 加入 $10 \%$ (质量比)单宁酸, 
促进微胶囊进一步交联和固化并保持缓慢搅拌(200 $\mathrm{r} / \mathrm{min}$ ) $30 \mathrm{~min}$. 然后, 加入蒸馏水稀释到 $400 \mathrm{~mL}$, 并利用 布氏漏斗(直径 $100 \mathrm{~mm}$ )过滤. 过滤物分别用水和异丙醇 各洗脱 2 次, 并置于真空干燥器内 $20 \sim 25^{\circ} \mathrm{C}$ 干燥 $24 \mathrm{~h}$. 最 后, 将干燥后所得到的微胶囊(5.95 g)过篮形成均匀的 粒径(100 150 $\mu \mathrm{m})$, 用样品瓶密封置于 $0 \sim 5^{\circ} \mathrm{C}$ 保存.

(iii) 微胶囊化产率的测定. 将 $10 \mathrm{mg}$ 微胶囊加 入 $2 \mathrm{~mL}$ 正己烷(含有 $20 \mu \mathrm{g} \mathrm{E}-10$-十六醛(内标))后, 通过超声波破碎仪(Soniprep 150, 间隔 $15 \mathrm{~s}$ )破囊 5 $\min ^{[22]}$. 样品微胶囊内包封的小菜蛾性信息素随之 溶解到正己烷中, 然后利用气相色谱进行定量测定. 气相色谱为HP5890- II 型, 色谱柱为DB-WAX极性柱 $(30 \mathrm{~m} \times 0.25 \mathrm{~mm} \times 0.2 \mu \mathrm{m})$, 载气为氮气, 无分流进 样, 通过FID检测器检测. 升温程序为: 起始温度 80 ${ }^{\circ} \mathrm{C}$ 保留 $5 \mathrm{~min}$, 然后以 $10^{\circ} \mathrm{C} / \mathrm{min}$ 升温至 $210^{\circ} \mathrm{C}$ 后保留 15 min. 微胶囊化产率按以下公式计算 [27]: 微胶囊 化产率 $=$ (微胶囊产品中芯材含量/原料中芯材含 量) $\times 100 \%$.

(iv) 田间释放保留率的测定. 将 60 个装有 10 $\mathrm{mg}$ 小菜蛾性信息素微胶囊的离心管 $(1.5 \mathrm{~mL}$, 盖子中 央有一直径为 $1 \mathrm{~cm}$ 的孔)放置在距诱捕实验田 $1 \mathrm{~km}$ 的 田间, 使其处于与田间诱捕实验相同的环境条件下 (温度、湿度以及风速等). 实验持续进行 6 周(2005 年 8 月 14 日 9 月 25 日), 最初间隔为 $2 \mathrm{~d}$, 随后间隔 为 $4 \mathrm{~d}$, 最后间隔为 $8 \mathrm{~d}$, 每次随机抽取 5 个离心管回

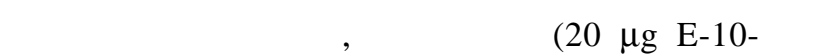
醛)的 $2 \mathrm{~mL}$ 正己烷提取, 并通过气相色谱测定微胶囊 中小菜蛾性信息素的残留量(条件同上). 微胶囊内的 小菜蛾性信息素保留率通过以下公式计算 ${ }^{[28]}$ : 保留 率 $=$ 释放后的微胶囊内芯材含量/初始释放时间微胶 囊内芯材含量) $\times 100 \%$.

（v）引诱活性的测定. 本实验在超大现代农业集 团(北京市平谷区)蔬菜基地的菜田中进行. 2005年 8 月 14 日 9 月 17 日的实验田为青花菜(Brassica oleracea L. var italica Plenck), 2006 年 6 月 6 日 7 月 6 日的实验田 为甘蓝 (Brassica oleracea $\mathrm{L}$. var capitata L.).

2005 年实验: 把装载 $30 \mathrm{mg}$ 微胶囊(当量为 1 个 橡胶诱芯)的离心管、橡胶诱芯(Z11-16:Ald, Z11-16:Ac 和 Z11-16:OH, 50:50:1)及空白橡胶诱芯固定在水盆诱 捕器(直径 $10 \mathrm{~cm}$ )中央, 距离水面 $1 \mathrm{~cm}$. 诱捕器放置在 青花菜上方 $30 \mathrm{~cm}$, 诱捕器间距为 $17 \mathrm{~m}$. 每个处理 5 次 重复. 实验期间, 每 $2 \mathrm{~d}$ 调查 1 次诱捕数量. 实验进行
5 周, 期间根据诱捕器实际情况进行换水.

2006 年实验: 为了检验咜存时间是否对微胶囊 的引诱效果具有影响, 用在 $0 \sim 5^{\circ} \mathrm{C}$ 条件下放置 1 年的 微胶囊(30 mg)和橡胶诱芯(“旧微胶囊”, “旧橡胶诱 芯”)与实验前合成的微胶囊(30 mg)和橡胶诱芯 (“新 微胶囊”, “新橡胶诱芯”)进行田间诱捕实验. 除未设 空白对照外, 与 2005 年引诱活性实验设置相同.

(vi) 迷向活性的测定. 2006 年 6 月 2 日 7 月 2 日, 在北京市平谷区的甘蓝田内进行了小菜蛾田间 迷向实验. 迷向处理田和化学农药防治处理田(对照) 间距为 $1 \mathrm{~km}$, 每个处理的实验面积均为 $0.02 \mathrm{hm}^{2}$, 各 设 5 个重复. 把装载 $30 \mathrm{mg}$ 小菜蛾性信息素微胶囊的 离心管绑到竹竿上, 使其距离地面 $1 \mathrm{~m}$, 竹竿间距为 $4 \sim 5 \mathrm{~m}$, 微胶囊施用量为 $18 \mathrm{~g} / \mathrm{hm}^{2}$. 为控制实验田内 的小菜蛾虫口密度, 实验前 (6 月 1 日)在迷向田和对 照田内利用“抑太保”(0.6 kg/hm²)进行了化学防治. 实验期间, 迷向田在第一个诱蛾高峰期后(6 月 9 日) 进行了 1 次Bt $\left(0.6 \mathrm{~L} / \mathrm{hm}^{2}\right)$ 防治, 对照田内利用“抑太 保”(0.6 kg/hm ${ }^{2}$ )进行了 4 次化学防治(6 月 9 日, 6 月 14 日, 6 月 20 日, 6 月 27 日). 在每块处理田中央均放置 1 个具有橡胶诱芯的诱捕器, 每 2 d调查 1 次诱捕数量. 迷向率通过以下公式计算: 迷向率 $=$ (对照田诱蛾数 迷向田诱蛾数 $) /$ 对照田诱蛾数 $\times 100 \%$.

(vii) 统计分析. 实验数据利用 SPSS11.01 进行 分析并通过 Tukey's 检验或 Paired-samples $t$ 检验进行 平均值间差异显著性分析 $(P<5 \%)$.

\section{2 结果与分析}

\section{1 微胶囊化产率}

由表 1 可见, 小菜蛾性信息素 3 种组分 Z1116:Ald, Z11-16: Ac 和 Z11-16: OH 的微胶囊化产率分 别为 $95.76 \%, 89.60 \%$ 和 $95.21 \%$, 说明小菜蛾性信息 素可以通过复合凝聚法较好地进行微胶囊化.

\section{2 田间释放保留率}

由图 1 可以看出, 在野外环境下(表 2)经过初始 $2 \mathrm{~d}$ 的快速释放后, 在随后的实验过程中, 微胶囊保 持了一个相对恒定的释放速率. 经过 6 周的释放, 微 胶囊内的Z11-16:Ald, Z11-16:Ac和Z11-16:OH的保 留率为 $5.52 \%, 6.81 \%$ 和 $10.63 \%$.

\section{3 引诱活性测定}

两年的诱捕实验结果表明, 在不同季节和不同虫 口密度下, 以微胶囊作为诱芯的诱捕器的引诱活性均 
表 1 小菜蛾性信息素的微胶囊化率

\begin{tabular}{ccccc}
\hline 信息素组分 & 样品微胶囊中的含量 $( \pm \mathrm{SE}) / \mu \mathrm{g}$ & 微胶囊中总含量 $( \pm \mathrm{SE}) / \mathrm{mg}$ & 初始重量 $/ \mathrm{mg}$ & 微胶囊化产率 $( \pm \mathrm{SE})(\%)$ \\
\hline Z11-16:Ald & $15.94 \pm 0.03$ & $9.48 \pm 0.01$ & 9.90 & $95.76 \pm 0.13$ \\
Z11-16:Ac & $14.9 \pm 0.02$ & $8.87 \pm 0.01$ & 9.90 & $89.60 \pm 0.08$ \\
Z11-16:OH & $0.32 \pm 0.01$ & $0.19 \pm 0.01$ & 0.20 & $95.21 \pm 0.01$ \\
\hline
\end{tabular}

表 2 实验期间的温度和相对湿度 $(2005 \text { 年 })^{\mathrm{a})}$

\begin{tabular}{|c|c|c|c|c|c|c|}
\hline \multirow{2}{*}{ 日期 } & \multicolumn{3}{|c|}{ 温度 $/{ }^{\circ} \mathrm{C}$} & \multicolumn{3}{|c|}{ 相对湿度(\%) } \\
\hline & 最高温度 & 最低温度 & 平均值 & 最高湿度 & 最低湿度 & 平均值 \\
\hline 8 月 $14 \sim 21$ 日 & 35.0 & 24.0 & 28.8 & 100.0 & 61.0 & 79.5 \\
\hline 8 月 $22 \sim 28$ 日 & 33.0 & 23.0 & 26.5 & 97.0 & 54.0 & 78.0 \\
\hline 8 月 29 日 9 月 4 日 & 33.0 & 21.0 & 25.7 & 94.0 & 47.0 & 76.0 \\
\hline 9 月 5 11日 & 32.0 & 19.0 & 23.4 & 96.0 & 57.0 & 75.0 \\
\hline 9 月 $12 \sim 18$ 日 & 30.0 & 14.0 & 21.6 & 91.0 & 52.0 & 73.0 \\
\hline 9 月 $19 \sim 26$ 日 & 29.0 & 13.0 & 20.3 & 89.0 & 40.0 & 68.5 \\
\hline
\end{tabular}

a) 实验期间天气，除 8 月 14 日夜间有小雨外，其余均为晴天

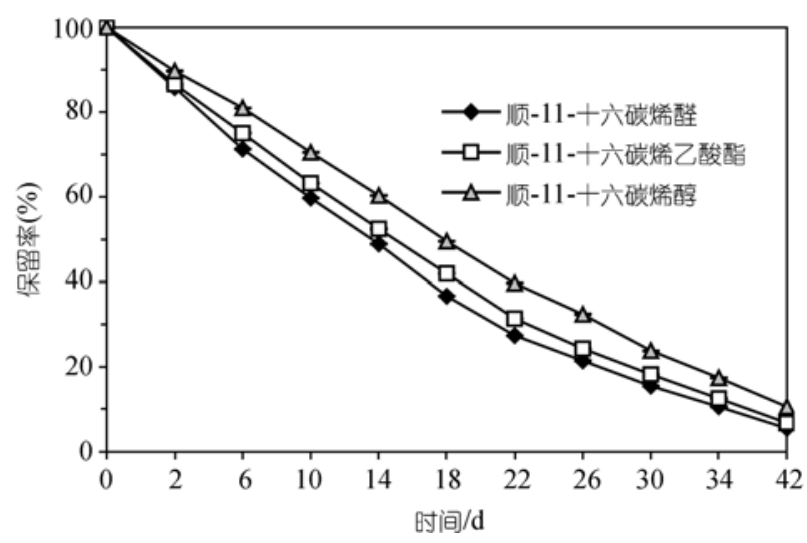

图 1 小菜蛾性信息素微胶囊的释放保留率(2005 年)

明显好于橡胶诱芯的引诱活性(图 2 和 3), 并且整个 实验期间微胶囊一直保持着对小菜蛾雄虫的引诱活 性(图 4 和 5). 2006 年的对比实验表明, 虽然经过咜存 的微胶囊与新合成的微胶囊在诱捕数量上有显著性 差异 $(P<0.05)$, 但是经过咜存的微胶囊的引诱活性 仍明显好于新合成的橡胶诱芯 $(P<0.05)$, 这说明在 咜存期间, 微胶囊比橡胶诱芯对小菜蛾性信息素提 供了更好的保护作用.

\section{4 迷向活性测定}

从表 3 可知, 在迷向处理前, 迷向处理田和对照 田内的小菜蛾虫口密度不存在显著性差异; 但经过 迷向处理后, 迷向田的诱捕数明显低于对照田的诱 捕数 $(P<0.05)$. 在实验期间, 迷向率维持在 $76.94 \%$ 98.67\%(表 3, 图 6).

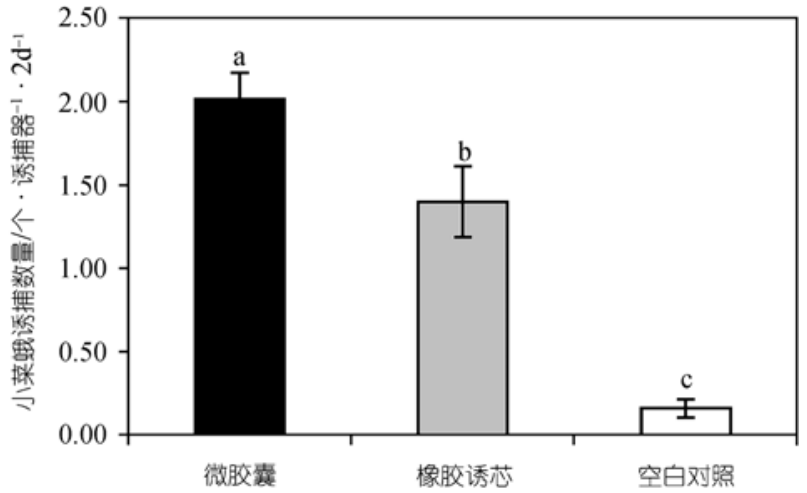

图 2 不同处理的小菜蛾诱捕数量比较(2005 年) 不同字母表示在 $5 \%$ 水平上差异显著

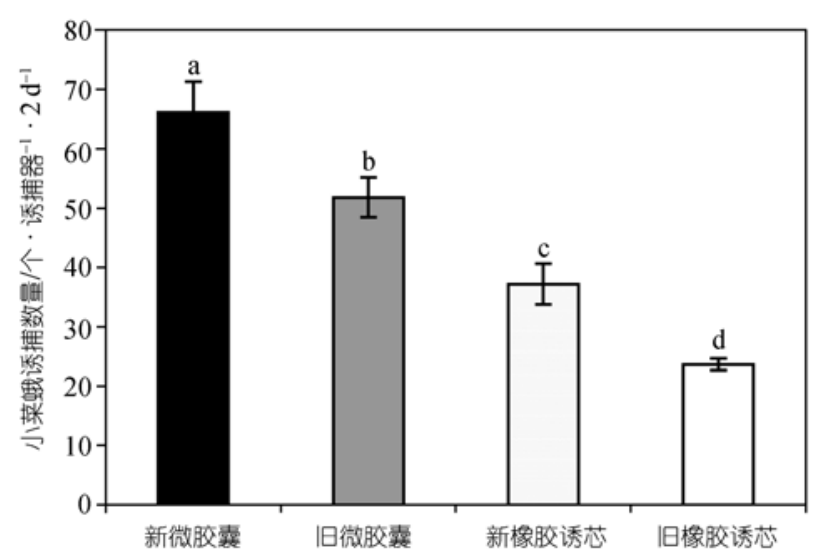

图 3 不同处理小菜蛾诱捕数量比较 (2006 年) 
表 3 迷向田和化学防治对照田中小菜蛾诱捕数量和迷向率 $(2006 \text { 年 })^{\mathrm{a})}$

\begin{tabular}{|c|c|c|c|}
\hline 日期 & $\begin{array}{c}\text { 迷向田诱捕数量 } \\
\text { (平均值 } \pm \text { 标准误差) }\end{array}$ & $\begin{array}{l}\text { 化防对照田诱捕数量 } \\
\text { (平均值 } \pm \text { 标准误差) }\end{array}$ & $\begin{array}{c}\text { 迷向率 }(\%) \\
\text { (平均值 } \pm \text { 标准误差) }\end{array}$ \\
\hline 6 月 2 日* & $22.20 \pm 2.23 \mathrm{a}$ & $21.30 \pm 3.46 \mathrm{a}$ & - \\
\hline 6 月 4 日 & $17.80 \pm 1.66 \mathrm{a}$ & $78.20 \pm 2.75 b$ & $76.94 \pm 2.68$ \\
\hline 6 月 6 日 & $21.20 \pm 0.97 \mathrm{a}$ & $105.20 \pm 11.56 \mathrm{~b}$ & $78.52 \pm 3.15$ \\
\hline 6 月 8 日 & $29.60 \pm 2.04 \mathrm{a}$ & $207.20 \pm 15.66 b$ & $85.41 \pm 1.39$ \\
\hline 6 月 10 日 & $15.80 \pm 1.53 \mathrm{a}$ & $134.60 \pm 12.20 \mathrm{~b}$ & $87.52 \pm 2.25$ \\
\hline 6 月 12 日 & $14.20 \pm 1.50 \mathrm{a}$ & $145.60 \pm 12.93 b$ & $89.86 \pm 1.69$ \\
\hline 6 月 14 日 & $7.20 \pm 1.88 \mathrm{a}$ & $96.40 \pm 4.74 \mathrm{~b}$ & $92.81 \pm 3.02$ \\
\hline 6 月 16 日 & $12.60 \pm 1.08 \mathrm{a}$ & $124.80 \pm 18.85 b$ & $89.02 \pm 1.60$ \\
\hline 6 月 18 日 & $7.60 \pm 0.68 \mathrm{a}$ & $72.20 \pm 8.05 b$ & $88.67 \pm 2.23$ \\
\hline 6 月 20 日 & $2.60 \pm 1.60 \mathrm{a}$ & $42.00 \pm 6.03 \mathrm{~b}$ & $94.62 \pm 3.49$ \\
\hline 6 月 22 日 & $3.60 \pm 1.50 \mathrm{a}$ & $37.20 \pm 6.92 b$ & $87.23 \pm 5.96$ \\
\hline 6 月 24 日 & $1.40 \pm 0.98 \mathrm{a}$ & $37.60 \pm 7.67 b$ & $96.54 \pm 2.12$ \\
\hline 6 月 26 日 & $0.80 \pm 0.37 \mathrm{a}$ & $23.80 \pm 3.51 \mathrm{~b}$ & $96.81 \pm 1.67$ \\
\hline 6 月 28 日 & $0.80 \pm 0.58 \mathrm{a}$ & $11.60 \pm 0.52 b$ & $93.46 \pm 4.85$ \\
\hline 6 月 30 日 & $1.20 \pm 0.37 \mathrm{a}$ & $17.60 \pm 2.48 b$ & $94.39 \pm 3.34$ \\
\hline 7 月 2 日 & $0.20 \pm 0.20 \mathrm{a}$ & $12.20 \pm 0.86 b$ & $98.67 \pm 1.33$ \\
\hline
\end{tabular}

a) $*$, 未处理前. 同行不同字母表示在 $5 \%$ 水平上差异显著(Paired-samples $t$ 检验)

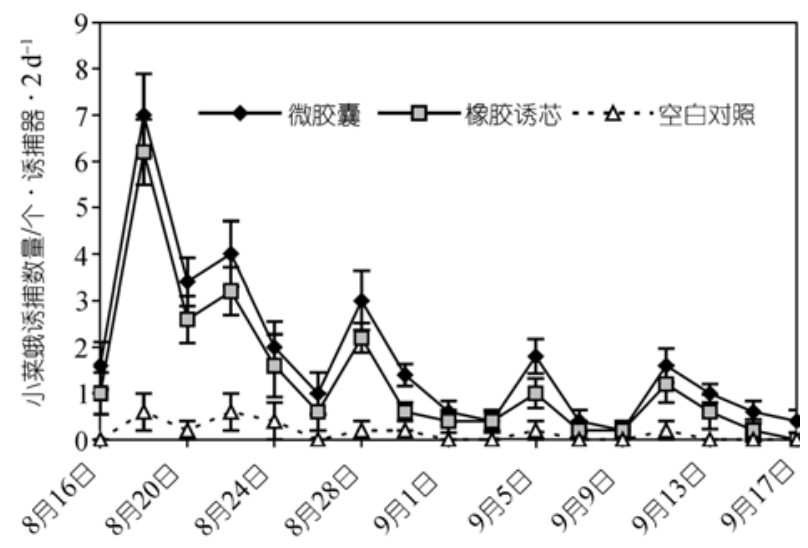

图 4 不同处理的小菜蛾诱捕趋势(2005 年)

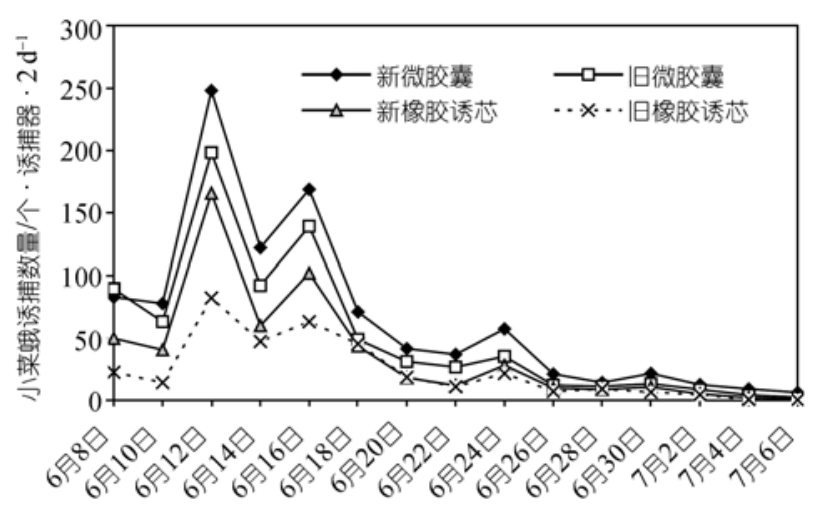

图 5 不同处理的小菜蛾诱捕趋势(2006 年)

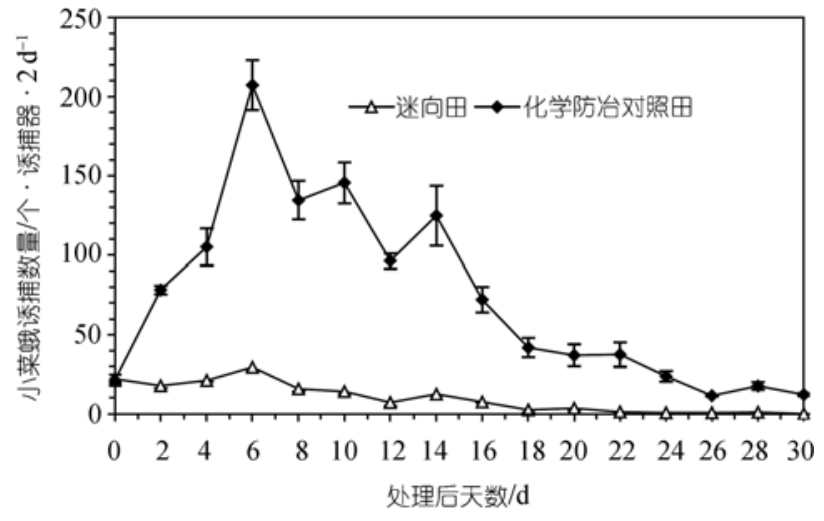

图 6 迷向田和化学防治对照田的小菜蛾诱捕趋势 (2006 年)

\section{3 讨论}

微胶囊化产率测定的结果表明, 以明胶和阿拉 伯树胶为壁材通过复合凝聚法可以有效地对小菜蛾 性信息素进行微胶囊化.

众所周知, 为了使微胶囊实现性能最优化, 其性 能评价应该在野外而不是在实验室中进行. 只有通过 田间实验测定微胶囊的释放速率、相对作用时间、空 间分布情况和天气因素的影响才能合理地对剂型进行 选择[23]. 在我们的田间实验中, 微胶囊保持了一个相 对恒定的释放速率. 并且在整个实验期间微胶囊均保 持了较好的引诱活性, 在相同的信息素浓度下, 微胶 
囊的诱捕效果明显好于橡胶诱芯的诱捕活性. 经过一 年 $\left(0 \sim 5^{\circ} \mathrm{C}\right)$ 咜存的微胶囊和橡胶诱芯在田间诱捕能力 均明显下降; 但旧微胶囊的田间诱捕活性仍明显好于 新橡胶诱芯的田间诱捕活性. 在迷向实验中, 迷向田 的小菜蛾诱捕数量明显少于化防对照田. 尽管为了商 业用途, 实验期间迷向田中平均每个诱捕器一天的小 菜蛾诱捕数量高于 8 头(经济阈值)时 ${ }^{[5]}$, 为压低小菜蛾 虫口密度, 在迷向田中用 $B t\left(0.6 \mathrm{~L} / \mathrm{hm}^{2}\right)$ 防治过 1 次, 在 对照田中利用“抑太保”(0.6 kg/hm²)进行了 4 次化学防 治, 然而在化防对照田中的小菜蛾诱捕数量仍明显高 于迷向田，这表明迷向田中的微胶囊有效的干扰了小 菜蛾雌雄虫间的性信息交流.

微胶囊通常是利用传统的施药器械在大面积范 围内施用, 并且可以通过调节微胶囊自身的一些参 数(微胶囊的壁厚、粒径的大小、囊壁材料以及囊芯 物质组成)来实现缓慢释放 ${ }^{[29]}$. 在我们的田间实验过 程中, 微胶囊是利用离心管作为载体进行释放的, 但 实验结果依然证明了小菜蛾性信息微胶囊具有较好 的缓释性能和引诱效果, 并且微胶囊合成过程相对 简便，在田间释放和咜存期间又能对性信息素提供 较好的保护并具有缓释功能, 因此微胶囊可作为一 种有效的小菜蛾性信息素释放载体在防治中应用.

本实验中使用的微胶囊为粒径为 $100 \sim 150 \mu \mathrm{m}$ 的 细小颗粒. 如果通过添加椂性材料使微胶囊能够黏 附于小菜蛾雄蛾体上, 这时携带了微胶囊的小菜蛾 雄虫, 其作用就会类似于静电 Entostat ${ }^{\mathrm{TM}}$ 粒子(http:// www.exosect.com/solutions/products/exosex.asp), 作为 一个飞行的性信息素释放载体发挥迷向作用. 因此, 很多工作还有待于进一步的深入展开, 从而使小菜 蛾性信息素微胶囊能够在将来的诱捕或迷向应用中 发挥更好的作用.

致谢 感谢赵成华研究员、路虹研究员、李明远研究员和 刘军经理为本实验提供的帮助.

\section{参 考 文 献}

1 Wang X P, Le V T, Fang Y L, et al. Trap affect on the capture of Plutella xylostella (Lepidoptera: Plutellidae) with sex pheromone lures in cabbage fields in Vietnam. Appl Entomol Zool, 2004, 39(2): 303-309[DOI]

2 Baker P B, Shelton A M, Andale J T. Monitoring of diamondback moth (Lepidoptera: Plutellidae) in cabbage with pheromones. J Econ Entomol, 1982, 75: 1025-1028

3 Shirai Y, Nakamura A. Relationship between the numbers of wild males captured by sex pheromone trap and the population density estimated from a mark-recapture study in the diamondback moth,
Plutella xylostella (L.) (Lepidoptera: Plutellidae). Appl Entomol Zool, 1995, 30: 543-549

4 Ronald T. Relationship between the number of males captured by sex pheromone traps and wild population density in the field. J Entomol Sci, 1997, 22: 120-137

5 Reddy G V, Guerrero A. Optimum timing of insecticide applications against diamondback moth Plutella xylostella in cole crops using threshold catches in sex pheromone traps. Manag Sci Pest, 2001, 57(1): 90-94[DOI]

6 Chisholm M D, Underhil E W, Steck W F. Field trapping of the diamondback moth (Lepidoptera: Plutellidae) using synthetic sex attractants. Environ Entomol, 1979, 8: 516-518

7 Mclaughlin J R, Mitchell E R, Kirsch P A. Mating disruption of DBM in cabbage: Reduction of mating and suppression of larval populations. J Econ Entomol, 1994, 97: 1198-1204

8 Reddy G V P, Urs K C D. Mass trapping of diamondback moth Plutella xylostella in cabbage fields using synthetic sex pheromone. Int Pest Cont, 1997, 39: 125-126

9 Reddy G V P, Guerrero A. Pheromone-based integrated pest management to control the diamondback moth Plutella xylostella in cabbage fields. Pest Manag Sci, 2000, 56: 882-888[DOI]

10 Chisholm M D, Underhill E W, Palaniswamy P, et al. Orientation disruption of male diamondback moths (Lepidoptera: Plutellidae) to traps baited with synthetic chemicals or female moths in small field plots. J Econ Entomol, 1984, 77: 157-160

11 Chow Y S. Disruption effect of the synthetic sex pheromone and its analogues on diamondback moth. In: Talker N S, ed. Management of Diamondback Moth and Other Crucifer Pests. Proceedings of the Second International Workshop. Taiwan: Asian Vegetable Research and Development Center (AVRDC) Publication, 1992. 105-108

12 Nemoto H, Yano E, Kiritani K. Pheromonal control of diamondback moth in the management of crufier pests. In: Talker N S, ed. Management of Diamondback Moth and Other Crucifer Pests. Proceedings of the Second International Workshop. Taiwan: Asian Vegetable Research and Development Center (AVRDC) Publication, 1992. 91-97

13 Ohbayashi N, Shimizu K, Nagata K. Control of diamondback moth using synthetic sex pheromones. In: Talker N S, ed. Management of Diamondback Moth and Other Crucifer Pests. Proceedings of the Second International Workshop. Taiwan: Asian Vegetable Research and Development Center (AVRDC) Publication, 1992. 99-104

14 Ohno T, Asayama T, Ichikawa K. Evaluation of communication disruption method using synthetic sex pheromone to suppress diamondback moth infestations. In: Talker N S, ed. Management of Diamondback Moth and Other Crucifer Pests. Proceedings of the Second International Workshop. Taiwan: Asian Vegetable Research and Development Center (AVRDC) Publication, 1992. 109-114

15 Chisholm M D. Field trapping of the diamondback moth (Lepidoptera: Plutellidae) using synthetic sex attractants. Environ Entomol, 1979, (8): 516-518

16 Chisholm M D, Steck W F, Underhill E W, et al. Field trapping of diamondback moth Plutella xylostella using an improved four-component sex attractant blend. J Chem Ecol, 1983, 9(1): 113-119[DOI]

17 Schroeder R M, Shelton A M, Ferguson C S, et al. Application of synthetic sex pheromone for management of diamondback moth, Pluttella xylostella, in cabbage. Entomol Exp Appl, 2000, 94: 
243-248[DOI]

18 Kawasaki K. Effects of ratio and amount of the two sex pheromonal components of the diamondback moth on male behavioral response. Appl Ent Zool, 1984, 19(4): 436-442

19 Testu A. Electroantennogram activities of sex pheromone analogues and their synergistic effect on diamondback moth in the field attraction. Appl Ent Zool, 1979,14(3): 362-364

20 Tamaki Y, Kawasaki K, Yamada H, et al. (Z)-11-hexadecenal and (Z)-11-hexadecenyl acetate: Sex pheromone components of the diamondback moth (Lepidoptera: Plutellidae). Appl Entomol Zool, 1977, 12(2): 208-210

21 Liu X, Macaulay E D M, Pickett J A. Propheromones that release pheromonal carbonyl compounds in light. J Chem Ecol, 1984, 10(5): 809-822[DOI]

22 Shulkin A, Stöver H D H. Polymer microcapsules by interfacial polyaddition between styrene-maleic anhydride copolymers and amines. J Memb Sci, 2002, 209: 421-432[DOI]

23 Caro J H, Freeman H P, Brower D L, et al. Comparative distribution and persistence of disparlure in woodland air after aerial application of three controlled-release formulations. J Chem Ecol, 1981, 7(5): 867-880[DOI]
24 Sanders C J, Weatherston I. Sex pheromone of the eastern spruce budworm: Optimum blend of trans- and cis-11-tetradecenal. Can Entomol, 1976, 108: 1285-1290

25 Campion D G, McVeigh L J, Hunter-Jones P, et al. Evaluation of microencapsulated formulations of pheromone components of the Egyptian cotton leafworm Spodoptera littoralis. In: Mitchell E R, ed. Management of Insect Pests with Semiochemicals: Concepts and Practice. New York: Plenum Press, 1981. 253-265

26 Hall D R, Marrs G J. Microcapsules. In: Jutsum A R, Gordon R F S, eds. Insect Pheromones in Plant Protection. New York: John Wiley \& Sons Ltd, 1989. 219-220

27 许时贞, 张晓鸣, 夏书芹, 等. 微胶囊技术一原理与应用. 北 京: 化学工业出版社, 2006. 149-150

28 Li Y H, Fan M W, Bian Z, et al. Chitosan-DNA microparticles as mucosal delivery system: Synthesis, characterization and release in vitro. Chin Med J, 2005, 118(11): 936-941

29 Hall D R, Nesbitt B F, Marrs G J, et al. Development of microencapsulated pheromone formulations. In: Leonhardt B A, Beroza M, eds. Insect Pheromone Technology: Chemistry and Applications. New York: American Chemical Society, 1981. 131-143 